\title{
Clinical and molecular characterization of Korean children with infantile and late-onset Pompe disease: 10 years of experience with enzyme replacement therapy at a single center
}

\begin{abstract}
Min-Sun Kim, MD', Ari Song, MD', Minji Im, MD', June Huh, MD, PhD', I-Seok Kang, MD', Jinyoung Song, MD, PhD', Aram Yang, MD², Jinsup Kim, MD ${ }^{3}$, Eun-Kyung Kwon ${ }^{4}$, Eu-Jin Choi', Sun-Ju Han ${ }^{5}$, Hyung-Doo Park, MD ${ }^{6}$, Sung Yoon Cho, MD, PhD', Dong-Kyu Jin, MD, PhD' ${ }^{1}$ Department of Pediatrics, Samsung Medical Center, Sungkyunkwan University School of Medicine, Seoul, ${ }^{2}$ Department of Pediatrics, Inha University College of Medicine, Incheon, ${ }^{3}$ Department of Pediatrics, Hanyang University College of Medicine, Seoul, ${ }^{4}$ Department of Pediatrics, Samsung Medical Center, Seoul, ${ }^{5}$ Samsung Biomedical Research Institute, Seoul, ${ }^{6}$ Department of Laboratory Medicine \& Genetics, Samsung Medical Center, Sungkyunkwan University School of Medicine, Seoul, Korea
\end{abstract}

Purpose: Pompe disease (PD) is an autosomal recessive disorder caused by a deficiency of acid alphaglucosidase resulting from pathogenic GAA variants. This study describes the clinical features, genotypes, changes before and after enzyme replacement therapy (ERT), and long-term outcomes in patients with infantile-onset PD (IOPD) and late-onset PD (LOPD) at a tertiary medical center.

Methods: The medical records of 5 Korean patients (2 male, 3 female patients) diagnosed with PD between 2002 and 2013 at Samsung Medical Center in Seoul, Republic of Korea were retrospectively reviewed for data, including clinical and genetic characteristics at diagnosis and clinical course after ERT. Results: Common initial symptoms included hypotonia, cyanosis, and tachycardia in patients with IOPD and limb girdle weakness in patients with LOPD. Electrocardiography at diagnosis revealed hypertrophic cardiomyopathy in all patients with IOPD who showed a stable disease course during a median follow-up period of 10 years. Patients with LOPD showed improved hepatomegaly and liver transaminase level after ERT.

Conclusion: As ERT is effective for treatment of PD, early identification of this disease is very important. Thus, patients with IOPD should be considered candidates for clinical trials of new drugs in the future.

Key words: Glycogen storage disease II, Alglucosidase alfa, Enzyme replacement therapy, GAA, Hypotonia

\section{Introduction}

Pompe disease (PD), also known as glycogen storage disease type II (GSD II), is caused by a deficiency of lysosomal acid alpha-glucosidase encoded by GAA. ${ }^{1)}$ This deficiency results in glycogen accumulation in various body systems such as the central nervous system, though it occurs most primarily in the muscle tissue. ${ }^{2)} \mathrm{PD}$ presents as a broad spectrum of clinical phenotypes and can be divided into 2 main groups by age of onset and progression of symptoms: infantile-onset PD (IOPD) and late-onset PD (LOPD). The prevalence of PD ranges from 1:14,000 to 1:300,000 depending on geographic region and ethnicity. ${ }^{3)}$ IOPD has an apparently higher incidence among African-Americans and Chinese, while LOPD appears to have a higher incidence in the Netherlands. ${ }^{4)}$

IOPD is the result of a near-complete deficiency of GAA, with an estimated frequency of 1 : $138,000 .{ }^{5)}$ IOPD is characterized by hypertrophic cardiomyopathy (HCMP) in $88 \%$ to $100 \%$
Corresponding author: Sung Yoon Cho, MD, PhD Department of Pediatrics, Samsung Medical Center, Sungkyunkwan University School of Medicine, 81 Irwon-ro, Gangnam-gu, Seoul 06351, Korea Tel: +82-2-6190-5227

Fax: +82-2-3410-0830

E-mail: nadri1217@naver.com https://orcid.org/0000-0003-2913-059X

Received: 30 August, 2018

Revised: 5 October, 2018

Accepted: 22 October, 2018
Copyright (c) 2019 by The Korean Pediatric Society

This is an open-access article distributed under the terms of the Creative Commons Attribution NonCommercial License (http://creativecommons.org/ licenses/by-nc/4.0/) which permits unrestricted noncommercial use, distribution, and reproduction in any medium, provided the original work is properly cited. 
of patients. ${ }^{6,7)}$ Symptoms develop at a median age of 18 months if left untreated and include cardiomegaly (92\%-96\%), congestive heart failure (50\%), respiratory distress (78\%), muscle weakness (63\%-96\%), and feeding difficulty (57\%). ${ }^{6}$ The disease can be rapidly progressive. Patients with IOPD who receive intervention and survive infancy have a high prevalence of weakness involving the oropharyngeal muscles, which can lead to difficulty with swallowing and speech. ${ }^{8)}$ If left untreated, patients typically die within one year. ${ }^{9,10)}$

LOPD is the result of a partial deficiency of GAA. The estimated prevalence is $1: 57,000 .{ }^{11)}$ Symptoms usually begin in the first decade of childhood or adulthood. In both children and adults, cardiac involvement is very rare, and symptoms are dominated by significant involvement of the skeletal muscles. ${ }^{4)}$ Patients with LOPD are characterized by limb girdle weakness; in addition, nonspecific clinical manifestations, including feeding intolerance, recurrent respiratory infection, skeletal deformity, consonant articulation disorder, and persistent diarrhea, should prompt the consideration of PD. The heart is usually not involved. In comparison with IOPD, LOPD is considered a "milder" phenotype, ${ }^{12)}$ likely due to higher residual enzyme activity, but there is still significant impact on morbidity, quality of life (QoL), and life expectancy. ${ }^{13)}$

Enzyme replacement therapy (ERT) for PD with recombinant human acid alpha-glucosidase was approved in both Europe and the United States in 2006. ERT has shown a positive impact on survival, motor development, and HCMP in patients with IOPD ${ }^{5,14)}$ and has prompted improvement of motor function and/or mobility and pulmonary function in adults with LOPD. ${ }^{2,15)}$ However, there are limited data on the effect of ERT in children with IOPD ${ }^{16)}$ Patients with IOPD usually show rapid progression of generalized muscle weakness, HCMP, and cardiorespiratory failure or respiratory infection causing death in the first 1 to 2 years of life. ${ }^{17)}$ Therefore, early diagnosis and application of ERT are important in patients with IOPD to increase the potential for survival.

This study comprehensively examined the clinical features, genotypes, and long-term outcomes in patients with PD following ERT.

\section{Materials and methods}

\section{Patients}

Five Korean patients ( 2 males and 3 females) diagnosed with PD between 2002 and 2013 at Samsung Medical Center in Seoul, Republic of Korea, were included in this study. All patients had been previously diagnosed by measurement of acid alpha-glucosidase activity in peripheral blood leukocytes or skeletal muscle biopsy specimens and by mutation analysis.

\section{Muscle biopsy}

Muscle biopsy specimens measuring approximately $1.5 \mathrm{~cm} \times 0.5$ $\mathrm{cm}$ were dissected through the skin with minimum trauma along the long axis of the fibers. Specimens were held at $-140^{\circ} \mathrm{C}$ for 10 to 30 seconds. Specimens were frozen in liquid nitrogen, and freshfrozen cryostat sections were prepared with hematoxylin and eosin, modified Gomori trichrome, oil red 0, periodic acid-Schiff (PAS), cresyl violet, and Sirius Red staining. Histochemical reactions were examined according to standard procedures. ${ }^{18)}$

\section{Molecular analysis}

Genomic DNA was isolated from peripheral blood leukocytes according to standard procedures. ${ }^{19)}$ Polymerase chain reaction was performed to amplify the entire coding region of exons 2 to 20 and the surrounding intronic sequences of the acid alpha-glucosidase gene, as described previously by Ko et al. ${ }^{20)}$

\section{Clinical evaluation}

Conventional doppler and 2-dimensional M-mode echocardiography were performed. According to the pediatric cardiomyopathy registry criteria, HCMP is defined as $z$ score $>2$ of LV wall thickness. pulmonary function testing (PFT) (in patients [P] 2 and 4), abdominal ultrasonography, skeletal survey, modified barium swallow testing, speech-language evaluation, Denver Developmental Screening Test, Korean-Wechsler Intelligence Scale For Children-Fourth Edition (K-WISC-IV), and Short Form 36 (SF-36v2) Health Survey were performed.

\section{Alpha-glucosidase IgG antibody measurement}

Alpha-glucosidase immunoglobulin G (IgG) antibody was measured with an enzyme-linked immunosorbent assay (ELISA). Antibody titer was the reciprocal of the highest sample dilution in the antibody that was detected by the ELISA.

\section{Manual muscle testing}

Manual muscle testing (MMT) is a highly reliable method for assessing strength and was used in all patients. During the test, the examiner stood to the side being evaluated, with the patient sitting upright and positioned to allow full movement of the joint against gravity. Grade 3 indicates no resistance, grade 4 indicates moderate resistance, and grade 5 indicates maximum resistance.

\section{Six-minute walk test}

The 6-minute walk test (6MWT) is practical and simple and requires a 100-foot hallway but no exercise equipment or advanced technician training. This test measures the distance that a patient can quickly walk along a flat, hard surface (e.g., the hallway) in six minutes. Most patients do not reach their maximum exercise capacity during the 6MWT. However, because most activities of daily living are performed at submaximal levels of exertion, the 6MWT may better reflect the functional exercise level for daily physical activities. ${ }^{21)}$ The test was performed on P2 and P4, as both could 
walk independently.

\section{Ethics statement}

This study was approved by the Institutional Review Board (IRB) of Samsung Medical Center, Sungkyunkwan University School of Medicine, Seoul, Republic of Korea (approval number: 2018-08-046) and was granted exemption of informed consent from IRB review.

\section{Results}

\section{Clinical characteristics of patients with PD at diagnosis}

Five patients ( 2 males and 3 females) with PD were included in this study (Table 1). Three patients (P1, P2, and P3) had IOPD, while 2 ( $\mathrm{P} 4$ and $\mathrm{P} 5$ ) had LOPD. Initial IOPD symptoms appeared at the age of 1 month in P1 and at the age of 5 months in P2 and P3. Initial LOPD symptoms appeared at the age of 5 years in $\mathrm{P} 4$ and at 1 year in P5. Common initial symptoms included hypotonia, cyanosis, and tachycardia in patients with IOPD and limb girdle weakness in patients with LOPD. All patients with IOPD showed HCMP at the time of diagnosis. P2, P3, and P5 were suspected to have PD after histologic examination of skeletal muscle due to hypotonia or muscle weakness (Figs. 1, 2). All diagnoses were confirmed based on acid alpha-glucosidase activity and genetic analysis (Table 2). Intravenous recombinant human alglucosidase alfa (Myozyme, Sanofi, Paris, France) has been available in Korea since 2007. Age at the start of ERT was 5 months in P1, 1 year in P2 and P3, 17 years 6 months in $\mathrm{P} 4$, and 6 years 2 months in $\mathrm{P} 5$.

\section{Enzyme activity and molecular analysis}

The acid alpha-glucosidase activity in all patients with PD had remarkably decreased below the normal range (Table 2). Sequencing of GAA revealed 9 previously reported pathogenic variants, including 2 nonsense and 5 missense mutations as well as 2 deletions (Fig. 3, Table 2). P1 and P5 had c.1822C>T (p.Arg608X), while P4 carried c.2238G >C (p.Trp746Cys), the most common LOPD mutation in Chinese patients. $^{22)}$

Table 1. Clinical characteristics of patients with infantile-onset Pompe disease and late-onset Pompe disease at diagnosis

\begin{tabular}{|c|c|c|c|c|c|}
\hline Variable & Patient 1, male & Patient 2, femal & Patient 3, female & Patient 4, female & Patient 5, male \\
\hline Gestational age & 38 wk 2 day & $41 w k$ & 37 wk 3 day & $39 w k$ & 39 wk 2 day \\
\hline \multicolumn{6}{|l|}{ Measurement at birth } \\
\hline Weight (kg) (percentile) & 4.3 (90th-97th) & 3.3 (50th) & 2.88 (25th) & 3.2 (50th) & 3.0 (25th-50th) \\
\hline Height (cm) (percentile) & 54 (97th) & 49 (50th) & 48 (25th-50th) & - & - \\
\hline Head circumference (cm) & 37 (90th-97th) & 33 (50th) & 33 (50th) & - & - \\
\hline Initial symptom & Tachycardia, cyanosis, hypotonia & Tachycardia, cyanosis & Motor developmental delay & Limb girdle weakness & Limb girdle weakness \\
\hline Age at diagnosis & $2 \mathrm{mo}$ & $9 \mathrm{mo}$ & $9 \mathrm{mo}$ & 17 yr 5 mo & 2 yr 9 mo \\
\hline Age at start of ERT & $5 \mathrm{mo}$ & $1 \mathrm{yr}$ & $1 \mathrm{yr}$ & 17 yr 6 mo & 6 yr 2 mo \\
\hline $\mathrm{C} / \mathrm{T}$ ratio & 0.65 & 0.67 & 0.65 & 0.5 & 0.5 \\
\hline HCMP (LVPM, EF) & Yes (14 mm, 28\%) & Yes (10.5 mm, 56.2\%) & Yes (10.5 mm, 19.2\%) & No (7.4mm, 75.4\%) & No (6.3 mm, 78.4\%) \\
\hline Hepatomegaly & No & Yes & Yes & Yes & Yes \\
\hline AST:ALT:CK & $319: 139: 5,804$ & 138:103:no data & 354:119:no data & $177: 139: 1067$ & 165:82:no data \\
\hline Ventilator support & Yes (1 mo) & No & Yes (10 yr) & No & Yes (5 yr 7 mo) \\
\hline Tracheostomy & Yes (6 mo) & No & Yes (10 yr 1 mo) & No & Yes (5 yr 7 mo) \\
\hline PFT & No data & Normal & No data & Restrictive pattern & No data \\
\hline Denver test & Motor, language delay & Normal & Motor, language delay & Normal & Motor delay \\
\hline Articulation disorder (\%) & No data & Yes (13.9) & Yes (31) & No data & Yes (69.7) \\
\hline Swallowing disorder & Yes & No & Yes & No & No \\
\hline Gastrostomy & Yes (2 yr 1 mo) & No & No & No & No \\
\hline Recurrent otitis media & Yes & No & Yes & No & No \\
\hline VT tube & Yes (4 yr 11 mo) & No & Yes (2 yr 1 mo) & No & No \\
\hline Hearing loss & Yes (1 yr 8 mo) & No & Yes (2 yr 1 mo) & No & No \\
\hline Others & FHx., renal stones, overgrowth & PSVT with WPW & Congenital cataract & Cleft palate & - \\
\hline
\end{tabular}

Gestational age and measurements were determined at birth. C/T ratio, HCMP on echocardiography, hepatomegaly on abdominal ultrasonography, and laboratory findings in patients with Pompe disease were determined at the time of diagnosis.

GA, gestational age; ERT, enzyme replacement therapy; C/T ratio, cardiothoracic ratio at initial symptom onset; HCMP, hypertrophic cardiomyopathy; LVPM, left ventricle posterior wall thickening; EF, ejection fraction; AST, aspartate aminotransferase; ALT, alanine aminotransferase; CK, creatine kinase; PFT, pulmonary function test; VT, ventilation tube; FHx, family history of Pompe disease; PSVT, paroxysmal supraventricular tachycardia; WPW, Wolff-Parkinson-White syndrome. 
LVWT (mm)

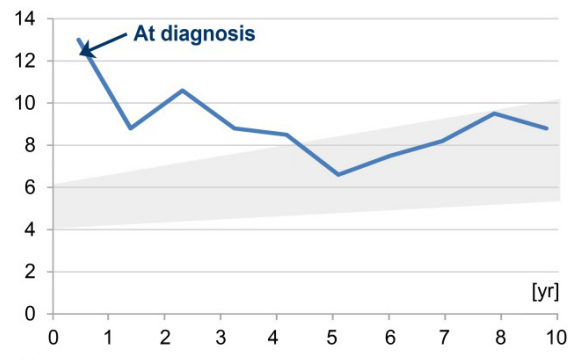

A

\section{LVWT (mm)}

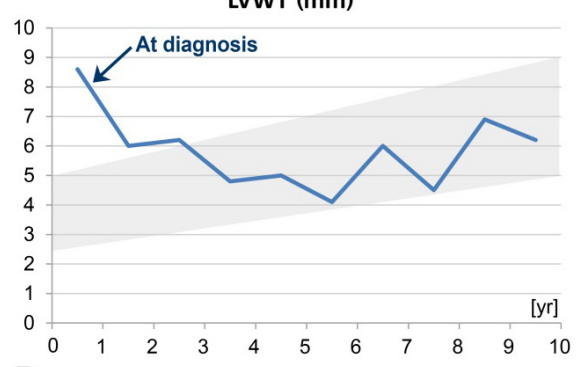

B

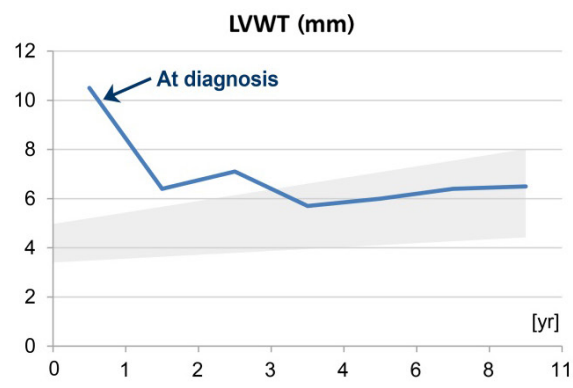

C
LV EF (\%)

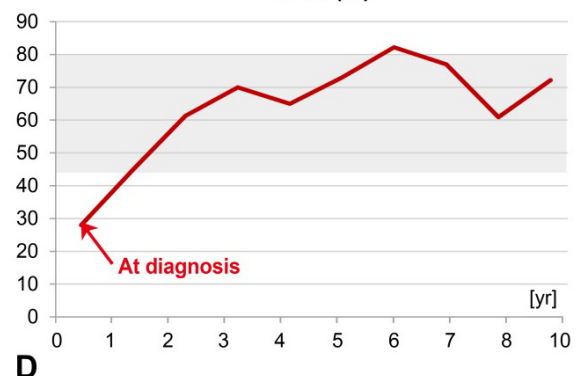

LV EF (\%)

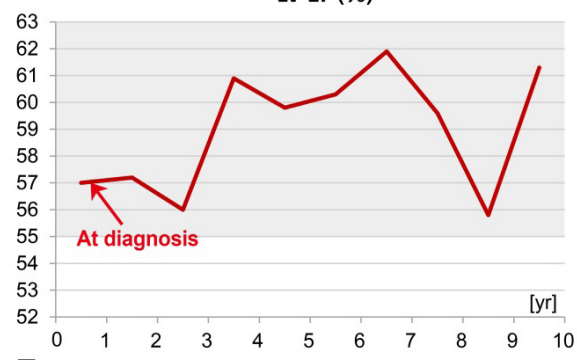

E

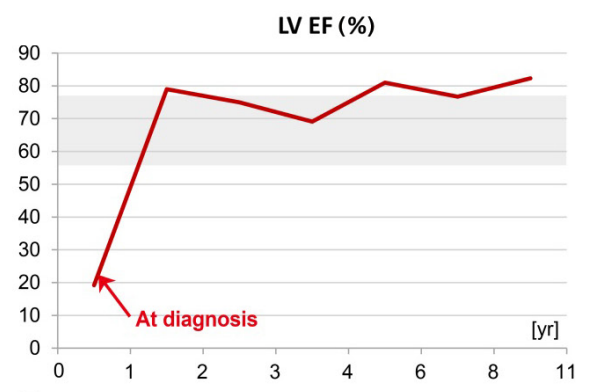

$\mathbf{F}$

Fig. 1. Changes in LVWT and LVEF after ERT in patients with IOPD. The gray background represents the normal range of LVWT and LVEF. LVWT and LVEF patient 1 (A, D), 2 (B, E), 3 (C, F). LVWT, left ventricle posterior wall thickness; LVEF, left ventricle ejection fraction; ERT, enzyme replacement therapy; IOPD, infantile-onset Pompe disease.
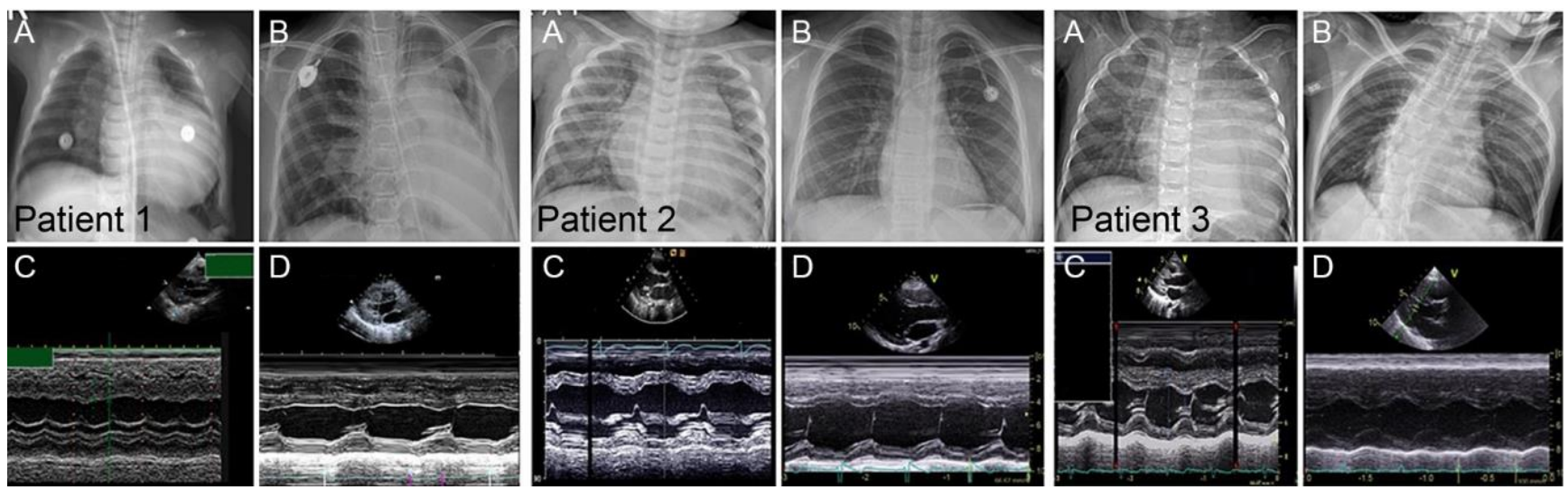

Fig. 2. Changes noted in chest radiography and 2-dimensional echocardiography images after ERT in a patient with IOPD cardiomegaly on chest X-ray before ERT (A) and after ERT (B); HCMP on 2-dimensional echocardiography before ERT (C) and after ERT (D). ERT, enzyme replacement therapy; IOPD, infantile-onset Pompe disease; HCMP, hypertrophic cardiomyopathy. 
Table 2. Enzyme activity analysis and gene mutations in patients with Pompe disease

\begin{tabular}{|c|c|c|c|c|c|c|c|c|c|}
\hline \multirow[t]{2}{*}{ Patient } & \multirow[t]{2}{*}{ Sex } & \multicolumn{3}{|c|}{$\begin{array}{l}\text { Acid } \alpha \text { glucosidase enzyme activity } \\
\text { (nmoL/hr/mgP) }\end{array}$} & \multirow[t]{2}{*}{ Site of biopsy } & \multirow[t]{2}{*}{ Genetic analysis } & \multirow{2}{*}{$\begin{array}{l}\text { Type of } \\
\text { mutation }\end{array}$} & \multirow[t]{2}{*}{ Exon } & \multirow[t]{2}{*}{ Reference } \\
\hline & & Activity & Specimen & Normal range & & & & & \\
\hline \multirow[t]{2}{*}{$1^{\text {a) }}$} & M & 5.4 & Leukocyte & $15.6 \pm 5.1$ & Skeletal muscle & c.875A>G (p.Tyr292Cys), & Missense & 3 & Park et al. ${ }^{33)} 2013$ \\
\hline & & & & & & c.1822C>T (p.Arg608X) & Nonsense & 13 & Fukuhara et al. ${ }^{30)} 2017$ \\
\hline \multirow[t]{2}{*}{$2^{\text {a) }}$} & $\mathrm{F}$ & 0.6 & Leukocyte & $15.6 \pm 5.1$ & Skeletal muscle & c.1316T>A (p.Met439Lys), & Missense & 8 & Park et al. ${ }^{33)} 2013$ \\
\hline & & & & & & c.1579_1580del (p.Arg527Glyfs*3) & Deletion & 16 & Ko et al. ${ }^{35)} 2018$ \\
\hline \multirow[t]{2}{*}{$3^{\text {a) }}$} & $\mathrm{F}$ & 5.1 & Leukocyte & $15.6 \pm 5.1$ & Skeletal muscle & c.2171C>A (p.Ala724Asp), & Missense & 14 & Cho et al. ${ }^{14)} 2012$ \\
\hline & & & & & & c.1322_1326+9del & Deletion & 7 & Cho et al. ${ }^{14)} 2012$ \\
\hline \multirow[t]{2}{*}{$4^{\text {b) }}$} & $\mathrm{F}$ & 3.8 & Leukocyte & $15.6 \pm 5.1$ & Liver & c.1156C>T (p.Arg386Cys), & Missense & 10 & Park et al. ${ }^{33)} 2013$ \\
\hline & & & & & & c.2238G>C (p.Trp746Cys) & Nonsense & 16 & Liu et al. ${ }^{22)} 2014$ \\
\hline \multirow[t]{2}{*}{$5^{\text {b) }}$} & M & 0.01 & Muscle & $0.2 \pm 1.0$ & Skeletal muscle & c.1309C>T (p.Arg437Cys), & Missense & 8 & Liu et al. ${ }^{22)} 2014$ \\
\hline & & & & & & c1822C>T (p.Arg608X) & Nonsense & 13 & Park et al. ${ }^{33)} 2013$ \\
\hline
\end{tabular}

a)Infantile-onset Pompe disease. 'bate-onset Pompe disease.

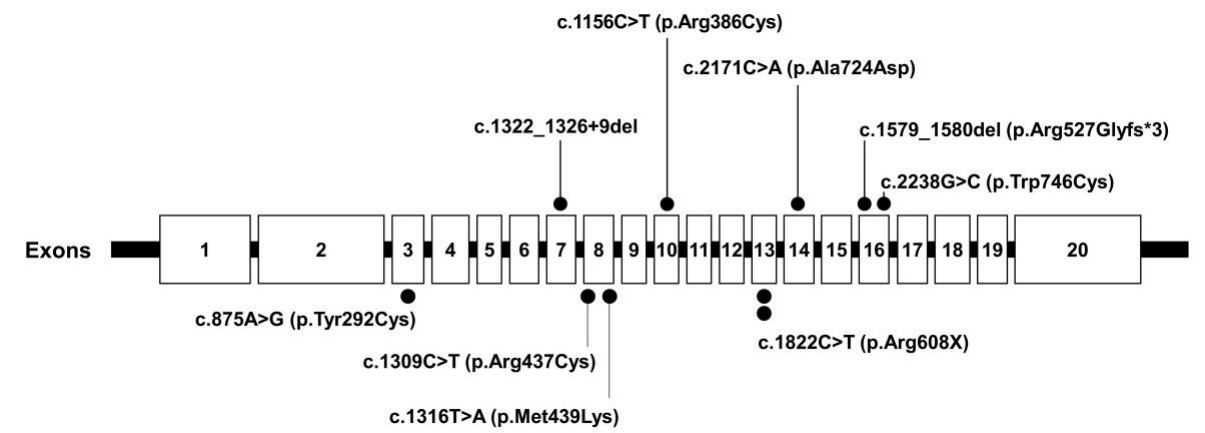

Fig. 3. Distribution of mutations in GAA in patients with Pompe disease.

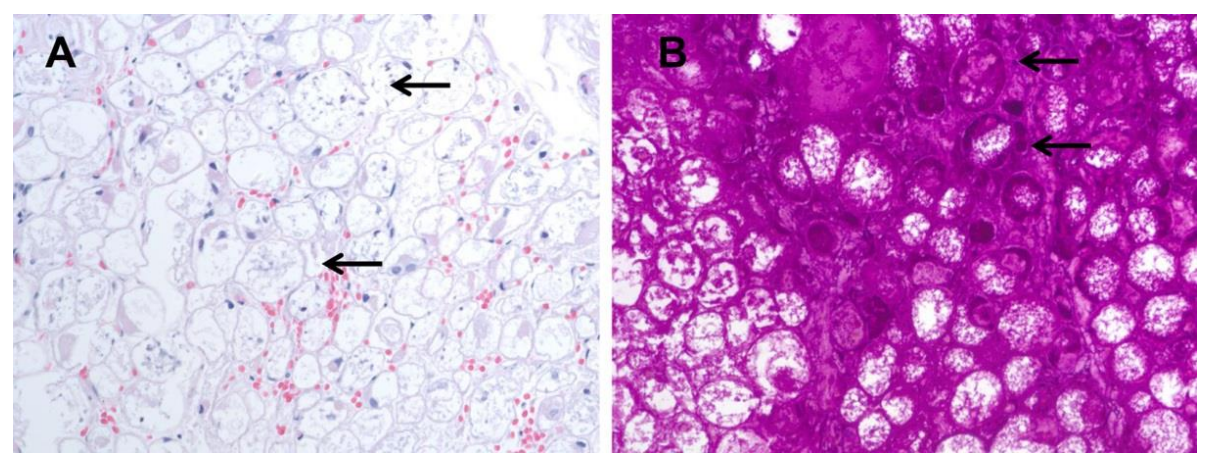

Fig. 4. Skeletal muscle biopsy in patient 1 with infantile-onset Pompe disease (IOPD). Thigh muscle biopsy performed at the age of 5 months in patient 1 with IOPD showed individual myofibers that were variably involved in multifocal or diffuse formations of glycogen pools, compressing and distorting the myofibrils in the cytoplasm. (A) Pronounced vacuolation of many fibers (arrows), some with very little red-staining myofibrillar material (H\&E, $\times 400)$. (B) Intense Periodic acid-Schiff staining of accumulated glycogen (arrows) $(\times 400)$.

\section{Pathologic findings}

All patients with IOPD had undergone muscle biopsy on suspicion of neuromuscular disease with hypotonia or muscle weakness before nine months of age. Histopathologic examination confirmed the presence of vacuolar changes in myofibers (glycogen-loaded lysosomes) that stained PAS-positive, and further testing for acid alpha-glucosidase was performed. These findings were present in the skeletal muscle of P1 (Fig. 4), P2, P3, and P5 and in the liver of P4. P4 underwent liver biopsy at the age of 7 years due to elevated liver enzymes and hepatomegaly. The liver biopsy showed incomplete PAS(+) fibrous septation on microscopic pathology and excessive accumulation of glycogen particles in the cytoplasmic matrix. Non- 
Table 3. Clinical response and laboratory findings with enzyme replacement therapy in patients with Pompe disease

\begin{tabular}{|c|c|c|c|c|c|c|c|c|c|c|c|c|c|c|c|}
\hline \multirow{2}{*}{ Patient } & \multicolumn{6}{|c|}{ Heart } & \multicolumn{4}{|c|}{ Lung } & \multicolumn{2}{|c|}{ Liver } & \multicolumn{2}{|c|}{$\begin{array}{c}\text { Muscle } \\
\text { weakness }\end{array}$} & \multirow{2}{*}{$\begin{array}{c}\text { Skeletal } \\
\text { deformity }\end{array}$} \\
\hline & \multicolumn{2}{|c|}{ Age } & $\begin{array}{c}\text { HCMP } \\
\text { (z score) }\end{array}$ & $\begin{array}{c}\text { LVPWT } \\
(\mathrm{mm})\end{array}$ & SF & $\begin{array}{l}\mathrm{EF} \\
(\%)\end{array}$ & Age & & VS & PFT & $\begin{array}{l}\text { Hepato- } \\
\text { megaly }\end{array}$ & LFT & $\begin{array}{l}\text { Wheel- } \\
\text { chair }\end{array}$ & CK & \\
\hline \multirow[t]{2}{*}{1} & At starting ERT & $5 \mathrm{mo}$ & Yes (18.73) & 14 & 12.6 & 28 & At diagnosis & $2 \mathrm{mo}$ & Yes & No data & No & $319 / 139$ & Yes & 5,804 & \multirow{2}{*}{$\begin{array}{l}\text { Scoliosis } \\
\text { Genu valgus } \\
\text { Knee- } \\
\text { contracture }\end{array}$} \\
\hline & After ERT & $5 \mathrm{yr}$ & No (1.45) & 7.5 & 50 & 82.2 & $\begin{array}{l}\text { At } 2 \text { yr after } \\
\text { ERT }\end{array}$ & $2 \mathrm{yr}$ & Yes & No data & No & 212/122 & Yes & 952 & \\
\hline \multirow[t]{2}{*}{2} & At starting ERT & $1 \mathrm{yr}$ & Yes (9.85) & 10.5 & 29 & 56.2 & At diagnosis & $9 \mathrm{mo}$ & No & No data & Yes & $138 / 103$ & No & No data & \multirow{2}{*}{ Genu valgus } \\
\hline & After ERT & $3 \mathrm{yr}$ & № (0) & 4.8 & 32.3 & 60.9 & $\begin{array}{l}\text { At } 2 \text { yr after } \\
\text { ERT }\end{array}$ & $3 \mathrm{yr}$ & No & Normal & No & $58 / 44$ & No & 358 & \\
\hline \multirow[t]{2}{*}{3} & At starting ERT & $1 \mathrm{yr}$ & Yes (9.85) & 10.5 & 8.7 & 19.2 & At diagnosis & $9 \mathrm{mo}$ & No & No data & Yes & $354 / 119$ & Yes & No data & \multirow{2}{*}{$\begin{array}{l}\text { Scoliosis } \\
\text { Planovalgus } \\
\text { Cavovarus }\end{array}$} \\
\hline & After ERT & $4 \mathrm{yr}$ & No (1.07) & 5.7 & 37.5 & 69.1 & $\begin{array}{l}\text { At } 2 \text { yr after } \\
\text { ERT }\end{array}$ & $3 \mathrm{yr}$ & Yes & No data & No & $112 / 105$ & Yes & 945 & \\
\hline \multirow[t]{2}{*}{4} & At starting ERT & 17 yr 6 mo & № (0.38) & 7.4 & 44 & 75.4 & At diagnosis & 17 yr 5 mo & No & SRP & Yes & $177 / 139$ & No & 1,067 & \multirow{2}{*}{ Scoliosis } \\
\hline & After ERT & $19 \mathrm{yr}$ & No (0.54) & 7.6 & 44.3 & 76.1 & $\begin{array}{l}\text { At } 2 \text { yr after } \\
\text { ERT }\end{array}$ & $19 \mathrm{yr}$ & No & SRP & No & $100 / 70$ & No & 877 & \\
\hline \multirow[t]{2}{*}{5} & At starting ERT & 6 yr 2 mo & No (1.87) & 6.3 & 40 & 78.4 & At diagnosis & 2 yr 9 mo & Yes & No data & Yes & $165 / 82$ & Yes & No data & \multirow{2}{*}{ Scoliosis } \\
\hline & After ERT & $8 \mathrm{yr}$ & № (1.88) & 6.8 & 45 & 77 & $\begin{array}{c}\text { At } 2 \text { yr after } \\
\text { ERT }\end{array}$ & $8 \mathrm{yr}$ & Yes & No data & No & $95 / 59$ & Yes & 610 & \\
\hline
\end{tabular}

ERT, enzyme replacement therapy; HCMP, hypertrophic cardiomyopathy; LVPWT, left ventricle posterior wall thickness; SF, shortening fraction; EF, ejection fraction; VS, ventilator support; PFT, pulmonary function testing; LFT, liver function test; CK, creatine kinase; SRP, severe restrictive pattern.

lysosomal type was observed on electron microscopy. Therefore, GSD III was suspected. However, the red blood cell glycogen assay was normal. Since the age of 10 years, $\mathrm{P} 4 \mathrm{had}$ had limb girdle weakness and could not walk or run well. At age 17 years, GAA sequencing revealed pathogenic mutations, and a PD diagnosis was made after confirmation of decreased acid alpha-glucosidase activity.

\section{Pulmonary function testing}

Two patients (P1 and P3) with IOPD and one patient (P5) with LOPD were ventilator-dependent. On PFT, P2 showed normal results. P4 showed a severe restrictive pattern (forced expiratory volume in 1 second/forced vital capacity $\left[\mathrm{FEV}_{1} / \mathrm{FVC}\right], 0.81 ; \mathrm{FVC}, 1.17$ $\mathrm{L} ; \mathrm{FEV}_{1}, 0.95 \mathrm{~L}$ ). Two patients (P1 and P3) with IOPD experienced recurrent respiratory infections, chronic otitis media, and hearing loss. P1 had respiratory distress from the first month of life and started receiving ventilator support. P3 was doing well without breathing difficulty despite repeated respiratory infections, but suddenly developed pneumonia after 9 years of ERT and subsequently required ventilator support due to respiratory failure. P5, who had LOPD, needed ventilator support from the time of diagnosis.

\section{Cardiac evaluation}

Three patients with IOPD showed HCMP without outflow tract obstruction (at the age of 2 months in P1 and 9 months in P2 and P3). P3 had moderate mitral regurgitation. These patients showed improvement of ejection fraction and left ventricular posterior wall thickening after ERT (Table 3, Figs. 1, 2). Two patients with LOPD have shown normal echocardiography to date.

\section{Hepatomegaly}

All patients showed elevated liver enzymes at diagnosis and a decrease in these levels with ERT. Four patients (P2, P3, P4, and P5) had hepatomegaly at diagnosis, but this was improved after ERT (Tables 1, 3).

\section{Skeletal deformity}

Four patients (P1, P3, P4, and P5) had scoliosis, and all patients with IOPD had valgus deformity mainly in the knee or ankle (Table 3, Fig. 5). P1 had knee contracture. Three patients (P1, P3, and P5) were wheelchair-dependent.

\section{Swallowing test}

P1 underwent gastrostomy and fundoplication at the age of 2 years due to gastroesophageal reflux and swallowing muscle weakness according to a modified barium swallow test. P3 had silent penetration before/during/after swallowing on the test at the age of 11 years and received frequent oral feedings in small amounts. No other patients had swallowing abnormalities (Table 1).

\section{Speech-language testing}

P2, P3, and P5 showed an articulation disorder in the pronunciation of consonants on speech-language evaluations. The accuracy of consonant pronunciation was expressed as a percentage (Table 1). P1 could not be evaluated due to tracheostomy and severe language developmental delay. 

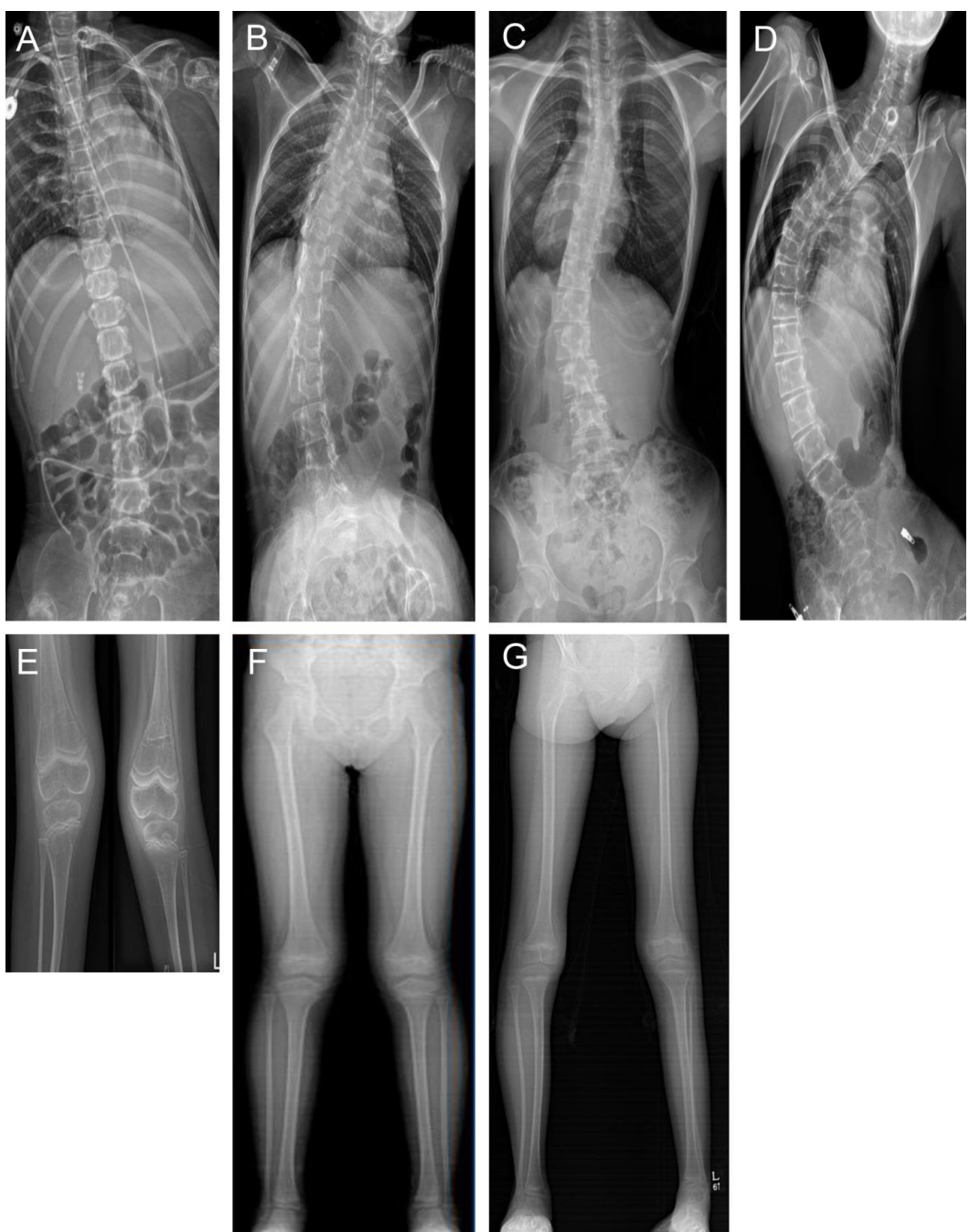

Fig. 5. Skeletal deformities in patients with Pompe disease (PD). Scoliosis in 4 patients with PD (i.e., patient 1, 3, 4, 5). Patient 1 (A), 3 (B), 4 (C), 5 (D), genu valgus and knee contracture in patient $1(\mathrm{E})$; genu valgus in patient $2(\mathrm{~F})$, right planovalgus and left cavovarus foot deformity in patient $3(G)$.

\section{Alpha-glucosidase immunoglobulin $\mathrm{G}$ antibody measurement}

Alpha-glucosidase IgG antibody levels of all patients were measured after 9 years (range, 5-11 years) of starting ERT. All 3 patients with IOPD had no alpha-glucosidase IgG antibody, while 2 patients with LOPD showed alpha-glucosidase IgG antibody level of 1,600 $\mathrm{U} / \mathrm{mL}$ in $\mathrm{P} 4$ and $200 \mathrm{U} / \mathrm{mL}$ in $\mathrm{P} 5$.

\section{Developmental delay}

Motor, personal-social, and language development was evaluated using the Denver Developmental Screening Test at diagnosis. The motor, personal-social, and language developmental levels in P1 were 5 months, 6 months, and 8 months, respectively, at 13 months. The developmental levels of P3 were 2 months, 5 months, and 8 months at the age of 15 months. P5 had normal language and personal-social development, but motor development was delayed to 20 months at the age of 10 years and eight months. P2 and P4 showed normal development at the time of diagnosis. The recent overall total intelligence of the P2, P4, and P5 measured by K-WISC-IV was 89 score (22.2th percentile), 86 score (18th percentile), 80 score (10th percentile), respectively, with lower scores than the average.

\section{Manual muscle testing}

P1 and P2 underwent MMT after 9 years of ERT. P1 only showed slight toe movement. P2 was unable to dorsiflex the ankles due to Achilles shortening, but the strength in the other muscles was good. P3 underwent MMT after 4 years and 11 years of ERT. Elbow strength showed no difference, and all other muscles showed reduced strength. P4 underwent MMT after 1 year and 5 years of ERT. In this patient, bilateral upper motor strength was grade 4 and above, but knee flexion/extension remained at grade 3/4; additionally, 
ankle dorsiflexion/plantarflexion increased from $4 / 4$ to $4 / 5$. P5 underwent MMT after 6 years and 12 years of ERT. Upper/lower motor strength decreased from $3 / 4$ to 2/3 (Table 4 ).

\section{Sort Form 36 Health Survey}

The SF-36v2 Health Survey uses 36 questions to measure functional status and was performed at the ages of 10 years 4 months in P1, 10 years 8 months in P2, 12 years 2 months in P3, 22 years 8 months in $\mathrm{P} 4$, and 18 years 3 months in $\mathrm{P} 5$ (Table 5). The physical component score (PCS) in P1, P3, and P5, who were wheelchairdependent, was less than 20 points, while the PCS in $\mathrm{P} 2$ and P4, who showed no progression of muscle weakness on MMT, was 70 points or more after ERT. In P2 with IOPD, ERT began at the age of 12 months, and this patient showed a higher total score for the survey, with a mental component score of 85.3125 and a PCS of 72.5 (Table 5).

\section{Side effects of ERT in patients with PD}

All patients continued to receive ERT without side effects, such as sweating, headaches, elevated temperature, or hypotension via infusions of recombinant human alglucosidase alfa. ${ }^{23)}$

Table 4. Results of manual muscle testing and 6-minute walk test in patients with Pompe disease

\begin{tabular}{|c|c|c|c|c|c|c|c|c|c|}
\hline \multirow{3}{*}{\multicolumn{2}{|c|}{ Variable }} & \multicolumn{8}{|c|}{ Patient (age at evaluation) } \\
\hline & & \multirow{2}{*}{$\frac{\mathrm{P} 1}{10 \mathrm{yr} 4 \mathrm{mo}}$} & \multirow{2}{*}{$\begin{array}{c}\mathrm{P} 2 \\
10 \mathrm{yr} 8 \mathrm{mo}\end{array}$} & \multicolumn{2}{|c|}{ P3 } & \multicolumn{2}{|c|}{ P4 } & \multicolumn{2}{|c|}{ P5 } \\
\hline & & & & 5 yr 8 mo & 12 yr 2 mo & 18 yr 5 mo & 22 yr 5 mo & 12 yr 2 mo & 18 yr $3 \mathrm{mo}$ \\
\hline \multicolumn{2}{|c|}{ Treatment period of ERT } & $9 \mathrm{yr}$ & $9 \mathrm{yr}$ & $4 \mathrm{yr}$ & $11 \mathrm{yr}$ & $1 \mathrm{yr}$ & $5 \mathrm{yr}$ & $6 \mathrm{yr}$ & $12 \mathrm{yr}$ \\
\hline \multirow[t]{2}{*}{ Shoulder } & Flexion & $0 / 0$ & $4 / 4$ & $4 / 4$ & $3 / 3$ & $4 / 4$ & $4 / 4$ & $4 / 4$ & $3 / 3$ \\
\hline & Extension & $0 / 0$ & $4 / 4$ & $4 / 4$ & $3 / 3$ & $4 / 4$ & $4 / 4$ & $4 / 4$ & $3 / 3$ \\
\hline \multirow[t]{2}{*}{ Elbow } & Flexion & $0 / 0$ & $5 / 5$ & $4 / 4$ & $4 / 4$ & $5 / 5$ & $5 / 5$ & $4 / 4$ & $3 / 3$ \\
\hline & Extension & $0 / 0$ & $5 / 5$ & $4 / 4$ & $4 / 4$ & $5 / 5$ & $5 / 5$ & $4 / 4$ & $3 / 3$ \\
\hline \multirow[t]{2}{*}{ Wrist } & Flexion & $0 / 0$ & $5 / 5$ & $4 / 4$ & $4 / 4$ & $5 / 5$ & $5 / 5$ & $4 / 4$ & $3 / 3$ \\
\hline & Extension & $0 / 0$ & $5 / 5$ & $4 / 4$ & $4 / 4$ & $5 / 5$ & $5 / 5$ & $4 / 4$ & $3 / 3$ \\
\hline \multirow[t]{2}{*}{ Finger } & Flexion & $0 / 0$ & $5 / 5$ & $4 / 4$ & $3 / 3$ & $5 / 5$ & $5 / 5$ & $4 / 4$ & $3 / 3$ \\
\hline & Extension & $0 / 0$ & $5 / 5$ & $4 / 4$ & $3 / 3$ & $5 / 5$ & $5 / 5$ & $4 / 4$ & $3 / 3$ \\
\hline \multirow[t]{2}{*}{ Hip } & Flexion & $0 / 0$ & $4 / 4$ & $4 / 4$ & $2 / 2$ & $2 / 2$ & $2 / 2$ & $3 / 3$ & $2 / 2$ \\
\hline & Extension & $0 / 0$ & $4 / 4$ & $3 / 3$ & $2 / 2$ & $2 / 2$ & $2 / 2$ & $3 / 3$ & $2 / 2$ \\
\hline \multirow[t]{2}{*}{ Knee } & Flexion & $0 / 0$ & $4 / 4$ & $4 / 4$ & $2 / 2$ & $3 / 3$ & $3 / 3$ & $3 / 3$ & $2 / 2$ \\
\hline & Extension & 0/0 & $5 / 5$ & $3 / 3$ & $2 / 2$ & $4 / 4$ & $4 / 4$ & $3 / 3$ & $2 / 2$ \\
\hline \multirow[t]{2}{*}{ Ankle } & Dorsiflexion & $0 / 0$ & $0 / 0$ & $3 / 3$ & $2 / 2$ & $4 / 4$ & $4 / 4$ & $3 / 3$ & $2 / 2$ \\
\hline & Plantarflexion & $0 / 0$ & $5 / 5$ & $4 / 4$ & $2 / 2$ & $4 / 4$ & $5 / 5$ & $3 / 3$ & $2 / 2$ \\
\hline \multirow[t]{2}{*}{ Toe } & Flexion & $1 / 1$ & $5 / 5$ & $4 / 4$ & $3 / 3$ & $5 / 5$ & $5 / 5$ & $3 / 3$ & $2 / 2$ \\
\hline & Extension & $1 / 1$ & $5 / 5$ & $4 / 4$ & $2 / 2$ & $5 / 5$ & $5 / 5$ & $3 / 3$ & $2 / 2$ \\
\hline \multicolumn{2}{|c|}{ 6-Minute walk test } & Not available & $343.29 \mathrm{mo}$ & Not available & - & \multicolumn{2}{|c|}{359.27 mo } & \multicolumn{2}{|c|}{ Not available } \\
\hline
\end{tabular}

Table 5. Evaluation of quality of life of patients with Pompe disease

\begin{tabular}{|c|c|c|c|c|c|}
\hline \multirow{2}{*}{ Short Form 36} & \multicolumn{5}{|c|}{ Patient (age at evaluation) } \\
\hline & P1 (10 yr 4 mo) & P2 (10 yr 8 mo) & P3 (12 yr 2 mo) & P4 (22 yr 8 mo) & P5 (18 yr 3 mo) \\
\hline Physical functioning & 0 & 45 & 0 & 55 & 0 \\
\hline Role limitations (physical) & 0 & 50 & 0 & 87.5 & 0 \\
\hline Bodily pain & 0 & 100 & 57.5 & 100 & 45 \\
\hline General medical health & 55 & 95 & 10 & 75 & 50 \\
\hline Vitality & 43.75 & 93.75 & 6.25 & 75 & 81.25 \\
\hline Social functioning & 62.5 & 625 & 12.5 & 100 & 100 \\
\hline Role limitations (emotional) & 0 & 100 & 0 & 100 & 100 \\
\hline Mental health & 45 & 85 & 40 & 70 & 95 \\
\hline Physical component score & 13.75 & 72.5 & 16.875 & 79.375 & 23.75 \\
\hline Mental component score & 37.8125 & 85.3125 & 14.6875 & 86.25 & 94.0625 \\
\hline
\end{tabular}




\section{Discussion}

This study examined clinical and molecular characteristics and the 10-year experience of ERT in 5 patients with IOPD and LOPD in a single center in Korea. Three patients (P1, P2, and P3) were diagnosed with IOPD and experienced initial symptoms by the age of 5 months. These individuals developed HCMP within 1 year after birth, but all have remained alive on ERT. Two patients (P4 and P5) were diagnosed with LOPD, and their initial symptom was limb girdle weakness. The most common initial symptom was cardiomegaly and muscle weakness in IOPD and LOPD, respectively. Cardiomegaly (100\% vs. 0\%) was found only in patients with IOPD, while hepatomegaly (66.7\% vs. 100\%) and muscle weakness (66.7\% vs. $100 \%$ ) were observed more frequently in patients with LOPD (Table 1). Respiratory assistance was required in $66.7 \%$ of IOPD patients and 50\% of LOPD patients, and wheelchairs were required in $60 \%$ of $\mathrm{PD}$ patients.

The GAA gene contains 20 exons and is highly polymorphic, with a large number of neutral variations. Thus far, 558 sequence variants of the GAA gene have been published in the Pompe Disease Mutation Database. The finding of some frequently occurring GAA mutations in certain populations points to single mutational events in those populations. The best-documented example is the African-American mutations, c.2560C >T (p.Arg854X). ${ }^{24)}$ A second well-known example of a founder mutation is c.1935C >A (p.ASP645Glu). ${ }^{25}$ c.1726G >A (p.Gly576Ser) and c.2065G >A (p.Glu689Lys) are more frequent among Asian populations. ${ }^{26,27)}$ These 2 sequence variants are most often found together on the same allele, and an estimated $3.3 \%$ to 3.9\% of people in Asian populations are homozygous for both variants. ${ }^{28)}$ c. $2481+102 \_2646+31$ del (deletion of exon18) is common in some subsets of the Caucasian population. ${ }^{29)}$ Some mutations appear with considerable frequency in particular ethnic groups. The c.546G>T (p.Thr182Phe) (22.9\%, 16 of 70) found to be the most common mutation, followed by c.1857C >G (p.Ala619Arg) (14.3\%, 10 of 70), in Japanese patients with PD. ${ }^{30)}$ The c. $1935 \mathrm{C}>\mathrm{A}$ (p.Asp645Glu) mutation accounted for 80\% (8 of 10 allele) of the GAA mutations found in the Thai population. ${ }^{31)}$ In Spanish patients with PD, the 2 most common GAA mutations, at frequencies of 25\% and 13.6\% respectively, were 11/44 allele (c.-32-13T>G,p.Leu705Pro) and 6/44 allele (c.1076-1G>C) ${ }^{32)}$ Additionally, the c. $1857 \mathrm{C}>\mathrm{G}$ (p.Ala619Arg) (23.3\%, 7 of 30 allele) and c.1316T>A (p.Met439Lys) (13.3\%, 4 of 30 allele) mutations were revealed as the most common in Korean PD patients. ${ }^{33)}$ In the present study, c. $1316 \mathrm{~T}>\mathrm{A}$ (p.Met439Lys) was detected in P2, who had IOPD. All PD patients had compound heterozygous mutations. Five missense mutations (c.875A >G (p.Tyr292Cys), c.1316G >A (p.Met439Lys), c.2171C>A (p.Ala724Asp), c.1156C>T (p.Arg386Cys), and Clinical and GAA gene mutation analysis in mainland Chinese patients (p.Arg437Cys)); 2 nonsense mutations (c.1822C>T (p.Arg608X) and c.2238G >C (p.Trp746Cys)); and 2 deletion mutations (c.1579_1580del
(p.Arg527Glyfs*3) and c.1322_1326+9del) were identified. No hotspot was found, and c.1822C >T (p.Arg608X) was found in 2 patients, specifically P1 (IOPD) and P5 (LOPD). P4 carried 2238G >C (p.Trp746Cys), the most common LOPD mutation found in Chinese patients. ${ }^{22)}$ c.1822C $>\mathrm{T}$ (p.Arg608X) was identified as the second-most common mutation considering only IOPD in Japanese patients. ${ }^{30)}$ c.32-13T>C (p.Leu705Pro) was the most common mutation when the first signs of the disease present before the age of $1{ }^{24)}$

ERT is not curative but does modify the disease course in patients with IOPD and LOPD. Patients with IOPD usually show rapid progression of the disease and die in the first 1 to 2 years of life without ERT. In this study, ERT was effective for improving survival, likely due to improvement of cardiomegaly and the thickness of the left ventricle in patients with IOPD (Table 3). Our patients with IOPD showed a stable disease course during a median of 10 years of follow-up. Early identification of IOPD is very important, as ERT increases lifespan and treats HCMP, hepatomegaly, and elevated liver transaminases despite persistent respiratory distress. Also, ERT was effective for treating hepatomegaly, elevated liver transaminase level, and to partially (only in P4) increase the strength of muscle in a patient with LOPD.

According to the existing literature, some patients develop infusion-associated reactions such as sweating, headaches, elevated temperature, or hypotension during infusions of recombinant human alglucosidase alfa. ${ }^{23)}$ However, in this study, all patients continued to receive ERT without side effects.

According to the existing literature, patients with PD have significantly lower QoL values in physical functioning, general health, vitality, and social functioning scales. ${ }^{34)}$ This study illustrated that, in patients (P2 and P4) with little muscle weakness and a lack of reliance on a wheelchair, QoL assessment revealed higher physical functioning and mental health component scores than in other patients. Interestingly, P5 had worsened muscle weakness and was dependent on a wheelchair, but he had the highest mental component score, even though his physical functioning score was low.

In PD, development of high titers of anti-GAA antibodies was primarily affected by cross-reactive immunologic material (CRIM) status. Therefore, CRIM status is an important predictor of response to ERT. In our study, none of the patients could be tested for CRIM status prior to ERT, but neutralizing antibodies that can influence the bioavailability of recombinant enzyme were measured. All 3 patients with IOPD had no alpha-glucosidase IgG antibody, while two patients with LOPD showed positive alpha-glucosidase IgG antibody level. Alpha-glucosidase IgG has been demonstrated to have a negative impact on ERT outcomes in one study. ${ }^{5)}$ Neutralizing antibodies developed in all treated patients, whether IOPD or LOPD. ${ }^{5)}$ IgG antibody titer showed peak level 12 weeks after ERT, and declined slowly thereafter. Higher infusion dose $(\mathrm{mg} / \mathrm{kg})$ was associated with higher antibody production. ${ }^{5)}$ However, IgG antibody titers did not correlate with treatment efficacy in other study. 
We will follow up alpha-glucosidase IgG antibody titers for all patients in this study.

In the present study, respiratory assistance was required in 60\% of PD patients. As impaired respiratory function is associated with high morbidity and impaired QoL, better treatments are needed. Alglucosidase alfa (Myozyme) is a recombinant human alphaglucosidase used for treatment of IOPD and LOPD. Several clinical trials and investigations for treatment of $\mathrm{PD}$ have been performed. To increase penetration into muscle cells and clear a greater substrate, new drugs are being developed. Neo-GAA (next-generation ERT) phase III trials are ongoing in naïve patients with LOPD in the United States and European Union. VAL-1221 (Valerion Therapeutics, Concord, MA, USA) is a therapeutic agent being considered in phase II clinical trials ongoing in patients with LOPD in the United Kingdom and the United States and is designed to deliver GAA to the cytoplasm and autophagic vacuoles as well as lysosomes. Duvoglustat coformulated with a proprietary ERT using the CHART Platform (Amicus Therapeutics, Cranbury, NJ, USA) is a nextgeneration small-molecule pharmacological chaperone that is part of a phase II trial ongoing in LOPD patients in Australia, Germany, the Netherlands, the United Kingdom, and the United States. Gene therapy using a recombinant adeno-associated viral vector (Audentes Therapeutics, San Francisco, CA, USA) is part of a phase I trial ongoing in patients with LOPD in the European Union and the United States.

The present study's limitations include the small number of patients and a lack of serial clinical parameters. However, in this study, the relatively long-term clinical course of IOPD was well-described, and these results indicate the effectiveness of ERT for IOPD. Therefore, patients with IOPD should be considered potential candidates for clinical trials of new drugs in the future.

\section{Conflicts of interest}

No potential conflict of interest relevant to this article was reported.

\section{Acknowledgments}

This study was supported by a grant from Samsung Medical Center (\#GF02170061).

\section{References}

1. Di Mauro S. Muscle glycogenoses: an overview. Acta Myol 2007;26: 35-41.

2. Case LE, Bjartmar C, Morgan C, Casey R, Charrow J, Clancy JP, et al. Safety and efficacy of alternative alglucosidase alfa regimens in
Pompe disease. Neuromuscul Disord 2015;25:321-32.

3. van der Ploeg AT, Reuser AJ. Pompe's disease. Lancet 2008;372:134253.

4. Vissing J, Lukacs Z, Straub V. Diagnosis of Pompe disease: muscle biopsy vs blood-based assays. JAMA Neurol 2013;70:923-7.

5. Chien YH, Hwu WL, Lee NC. Pompe disease: early diagnosis and early treatment make a difference. Pediatr Neonatol 2013;54:219-27.

6. van den Hout HM, Hop W, van Diggelen OP, Smeitink JA, Smit GP, Poll-The BT, et al. The natural course of infantile Pompe's disease: 20 original cases compared with 133 cases from the literature. Pediatrics 2003;112:332-40.

7. Dragulescu A, Mertens L, Friedberg MK. Interpretation of left ventricular diastolic dysfunction in children with cardiomyopathy by echocardiography: problems and limitations. Circ Cardiovasc Imaging 2013;6:254-61.

8. van Gelder CM, van Capelle CI, Ebbink BJ, Moor-van Nugteren I, van den Hout JM, Hakkesteegt MM, et al. Facial-muscle weakness, speech disorders and dysphagia are common in patients with classic infantile Pompe disease treated with enzyme therapy. J Inherit Metab Dis 2012;35:505-11.

9. Hirschhorn R, Reuser AJ. Glycogen storage disease type II: acid $\alpha$ glucosidase (acid maltase) deficiency. In: Scriver CR, Beaudet AL, Sly WS, Valle D, editors. The metabolic and molecular basis of inherited disease. 8th ed. New York: McGraw-Hill, 2001:3389-420.

10. Raben N, Plotz P, Byrne BJ. Acid alpha-glucosidase deficiency (glycogenosis type II, Pompe disease). Curr Mol Med 2002;2:145-66.

11. Ausems MG, Verbiest J, Hermans MP, Kroos MA, Beemer FA, Wokke $\mathrm{JH}$, et al. Frequency of glycogen storage disease type II in The Netherlands: implications for diagnosis and genetic counselling. Eur J Hum Genet 1999;7:713-6.

12. Van der Beek NA, Hagemans ML, Reuser AJ, Hop WC, Van der Ploeg AT, Van Doorn PA, et al. Rate of disease progression during longterm follow-up of patients with late-onset Pompe disease. Neuromuscul Disord 2009;19:113-7.

13. Gungor D, de Vries JM, Hop WC, Reuser AJ, van Doorn PA, van der Ploeg AT, et al. Survival and associated factors in 268 adults with Pompe disease prior to treatment with enzyme replacement therapy. Orphanet J Rare Dis 2011;6:34.

14. Cho A, Kim SJ, Lim BC, Hwang H, Park JD, Kim GB, et al. Infantile Pompe disease: clinical and genetic characteristics with an experience of enzyme replacement therapy. J Child Neurol 2012;27:319-24.

15. de Vries JM, van der Beek NA, Hop WC, Karstens FP, Wokke JH, de Visser M, et al. Effect of enzyme therapy and prognostic factors in 69 adults with Pompe disease: an open-label single-center study. Orphanet J Rare Dis 2012;7:73.

16. Strothotte S, Strigl-Pill N, Grunert B, Kornblum C, Eger K, Wessig C, et al. Enzyme replacement therapy with alglucosidase alfa in 44 patients with late-onset glycogen storage disease type 2: 12-month results of an observational clinical trial. J Neurol 2010;257:91-7.

17. Kishnani PS, Hwu WL, Mandel H, Nicolino M, Yong F, Corzo D, et al. A retrospective, multinational, multicenter study on the natural history of infantile-onset Pompe disease. J Pediatr 2006;148:671-6.

18. Werneck LC, Lorenzoni PJ, Kay CS, Scola RH. Muscle biopsy in Pompe disease. Arq Neuropsiquiatr 2013;71:284-9.

19. Hermans MM, van Leenen D, Kroos MA, Reuser AJ. Mutation detection in glycogen storage-disease type II by RT-PCR and automated sequencing. Biochem Biophys Res Commun 1997;241:414-8.

20. Ko TM, Hwu WL, Lin YW, Tseng LH, Hwa HL, Wang TR, et al. Molecular genetic study of Pompe disease in Chinese patients in Taiwan. Hum Mutat 1999;13:380-4.

21. ATS Committee on Proficiency Standards for Clinical Pulmonary Function Laboratories. ATS statement: guidelines for the six-minute 
walk test. Am J Respir Crit Care Med 2002;166:111-7.

22. Liu X, Wang Z, Jin W, Lv H, Zhang W, Que C, et al. Clinical and GAA gene mutation analysis in mainland Chinese patients with late-onset Pompe disease: identifying c.2238G > C as the most common mutation. BMC Med Genet 2014;15:141.

23. Chen M, Zhang L, Quan S. Enzyme replacement therapy for infantileonset Pompe disease. Cochrane Database Syst Rev 2017;11:CD011539.

24. Kroos M, Hoogeveen-Westerveld M, van der Ploeg A, Reuser AJ. The genotype-phenotype correlation in Pompe disease. Am J Med Genet C Semin Med Genet 2012;160C:59-68.

25. Lin CY, Shieh JJ. Molecular study on the infantile form of Pompe disease in Chinese in Taiwan. Zhonghua Min Guo Xiao Er Ke Yi Xue Hui Za Zhi 1996;37:115-21.

26. Tajima Y, Matsuzawa F, Aikawa S, Okumiya T, Yoshimizu M, Tsukimura T, et al. Structural and biochemical studies on Pompe disease and a "pseudodeficiency of acid alpha-glucosidase". J Hum Genet 2007;52:898-906.

27. Huie ML, Menaker M, McAlpine PJ, Hirschhorn R. Identification of an E689K substitution as the molecular basis of the human acid alphaglucosidase type 4allozyme (GAA*4). Ann Hum Genet 1996;60:3658.

28. Kroos MA, Mullaart RA, Van Vliet L, Pomponio RJ, Amartino H, Kolodny EH, et al. p.[G576S; E689K]: pathogenic combination or polymorphism in Pompe disease? Eur J Hum Genet 2008;16:875-9.

29. Dagnino F, Stroppiano M, Regis S, Bonuccelli G, Filocamo M. Evi- dence for a founder effect in Sicilian patients with glycogen storage disease type II. Hum Hered 2000;50:331-3.

30. Fukuhara Y, Fuji N, Yamazaki N, Hirakiyama A, Kamioka T, Seo JH, et al. A molecular analysis of the GAA gene and clinical spectrum in 38 patients with Pompe disease in Japan. Mol Genet Metab Rep 2017; 14:3-9.

31. Amarinthnukrowh P, Tongkobpetch S, Kongpatanayothin A, Suphapeetiporn K, Shotelersuk V. p.D645E of acid $\alpha$-glucosidase is the most common mutation in thai patients with infantile-onset pompe disease. Genet Test Mol Biomarkers 2010;14:835-7.

32. Gort L, Coll MJ, Chabas A. Glycogen storage disease type II in Spanish patients: high frequency of c.1076-1G>C mutation. Mol Genet Metab 2007;92:183-7.

33. Park HD, Lee DH, Choi TY, Lee YK, Lee SY, Kim JW, et al. Three patients with glycogen storage disease type II and the mutational spectrum of GAA in Korean patients. Ann Clin Lab Sci 2013;43:3116.

34. Hagemans ML, Laforet P, Hop WJ, Merkies IS, Van Doorn PA, Reuser AJ, et al. Impact of late-onset Pompe disease on participation in daily life activities: evaluation of the Rotterdam Handicap Scale. Neuromuscul Disord 2007;17:537-43.

35. Ko JM, Park KS, Kang Y, Nam SH, Kim Y, Park I, et al. A new integrated newborn screening workflow can provide a shortcut to differential diagnosis and confirmation of inherited metabolic diseases. Yonsei Med J 2018;59:652-61. 